\title{
Experimental and theoretical study of stimulated Raman scattering indicatrix asymmetry
}

\author{
M.V. Dmitriev, O.Iu. Isaienko, A.I. Ivanisik, P.A. Korotkov \\ Taras Shevchenko Kyiv National University \\ 4g, Academician Glushkov Avenue, 03127 Kyiv, Ukraine \\ Phone: +380-44-526-0570; e-mail:aivan@univ.kiev.ua
}

\begin{abstract}
The paper provides experimental and theoretical results of stimulated Raman scattering Stokes component indicatrix asymmetry study. Theoretical results demonstrate that asymmetry is caused by influence of Stokes/anti-Stokes parametric processes. Stokes/anti-Stokes parametric process provides additional photons to the forward Stokes component. This excess of photons compared to a backward Stokes component increases due to combinational mechanism of stimulated scattering during further propagation of waves along the medium. Thus, under competition of forward and backward Stokes components generation, the process of forward stimulated Raman scattering takes significant advantage. Obtained in this paper is satisfactory matching between theoretical calculations and experimental results for benzene under excitation by laser pulses with the duration $25 \mathrm{~ns}$ and wavelength $694.3 \mathrm{~nm}$.
\end{abstract}

Keywords: stimulated Raman scattering, parametric process, indicatrix.

Manuscript received 30.11.11; revised version received 29.12.11; accepted for publication 26.01.12; published online 29.02.12.

\section{Introduction}

According to Placzek's theory [1], the cross-section of Raman scattering that propagates along the exciting beam is the same in both forward and backward directions. However, experimental observations of spontaneous scattering sometimes show slight asymmetry [2]. Furthermore, in case of stimulated Raman scattering (SRS) Stokes component this asymmetry is essential in almost all experimental conditions. Intensity of the forward SRS first Stokes component is usually much higher than the intensity of the backward scattering, which propagates in the opposite direction with respect to the exciting laser beam. However, the nature of SRS indicatrix asymmetry is still controversial.

Many physical mechanisms explaining the SRS indicatrix asymmetry have been proposed. One of the earliest views was based on the fact that damping of coherent molecular vibrations wave generated by forward scattering is less than damping of the same wave generated by backward scattering, because its wave vector in the first case is much higher. Due to this difference in damping, scattering mostly propagates in the forward direction [3]. But this view didn't get any further evidences. A similar situation has developed with the following statement. Under the forward scattering, field transfers less momentum to molecules than that in case of backward scattering, therefore such different recoil effects substantially amplify the forward scattering [4].

Other views are more fundamental. In particular, attention has been drawn to the fact that Raman amplification in the forward direction is inversely proportional to the width of the spontaneous Raman scattering line. Raman amplification in the backward direction is less intensive, because it is inversely proportional to the total width of the spontaneous scattering line and the width of the exciting radiation spectrum [5]. Obviously, this mechanism is significant only in those rare cases where the width of exciting radiation spectrum is comparable to the width of the SRS line.

For ultra-short pulses, the length of the forward scattering path is limited by the SRS-active medium length, and the length of backward scattering is limited by the pulse length. Under these conditions forward scattering dominates [5].

Due to the change of refractive index during medium excitation pulse, phase modulation of interacting waves occurs, primarily improving the conditions for backward scattering [6].

Scattering occurs mainly in the focal region (focusing or self-focusing), and rest of the medium acts as an amplifier. If the focal region location does not coincide with the medium center, then the scattering will have a corresponding asymmetry [5]. 
There are acceptable and proven views that describe backward asymmetry. For example, amplification of backward SRS pulses occurs in the field of non-depleted exciting radiation thus leading to dominating backward scattering [7].

There is another approach that seems to be logical, but controversal. Due to interaction of exciting radiation and the forward Stokes wave by parametric mechanism, anti-Stokes component is generated. The energy outflow restrains forward Stokes wave amplification. In case of perfect phase matching between Stokes and anti-Stokes waves, none of them is able to exponentially amplify, and so parametric suppression of forward scattering takes place. As for the backward scattering, matching conditions for parametric process are not met in the described environment. As a result, dominating forward Stokes wave is generated $[5,8]$.

The aim of this work is experimental and theoretical study of Stokes/anti-Stokes parametric process influence on the SRS Stokes component indicatrix asymmetry. It is interesting, because forward and backward SRS are widely used in fiber Raman amplifiers [9].

\section{Experimental study}

Our experiment consisted of simultaneous energy registration in forward and backward SRS pulses. The experimental setup has been presented in Fig. 1.

$\mathrm{Al}_{2} \mathrm{O}_{3}: \mathrm{Cr}^{3+}$ crystal laser worked on one longitudinal mode, generating radiation pulses with duration $25 \mathrm{~ns}$, wavelength $694.3 \mathrm{~nm}$, spectral width $\leq 0.01 \mathrm{~cm}^{-1}$, energy $\sim 0.6 \mathrm{~J}$, divergence $5^{\prime}$ (arcmin). Laser radiation was damped using calibrating light filters and collimated to the center of the cuvette (length $L=1 \mathrm{~m}$ ) using the lens with the focal length $f=1 \mathrm{~m}$, cuvette was benzene-filled (active molecular vibrations are at $992 \mathrm{~cm}^{-1}$ ). The light, scattered in forward and backward directions, passed through blue-green filters BGF20 that completely absorb laser radiation, and directed to calorimetric energy meters of IMO-2N type with sensitivity $\geq 0.1 \mathrm{~mJ}$. Particular attention was paid to defining the relevant sensitivities that both energy meters provide on forward and backward scattering channels. An optical scheme based on equal division of laser beam was constructed to achieve this goal.

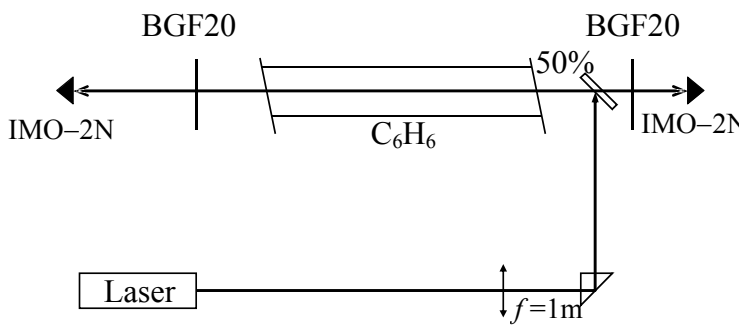

Fig. 1. The experimental setup.
To avoid the influence of parasitic link between the cuvette and laser caused by stimulated BrillouinMandelstam scattering (SBMS), dual optical distance between the lens focal point in the cuvette and laser was chosen at $\geq 4.5 \mathrm{~m}$, which corresponds to the timed feedback delay $\geq 15 \mathrm{~ns}$. In addition, the $50 \%$ mirror was placed on the path between laser and the cuvette, which further lowered efficiency of "SBMS mirror" by 4 times, even without taking into account the light attenuation filters (that were used at low excitation levels) and losses on other optical elements.

Non-parallelism of cuvette windows between each other and their non-perpendicularity to laser beam exceeded $5^{\circ}$. Other optical elements deviated from the normal at the angle $\sim 1^{\circ}$.

The peak intensity of radiation was calculated at the cuvette inlet for similar to Gaussian laser pulses with the maximum energy $\mathbf{E}_{L 0}=310 \mathrm{~mJ}$, duration $25 \mathrm{~ns}$, average beam radius $3.4 \mathrm{~mm}$ at $1 / \mathrm{e}$ level, and the obtained result was $I_{L 0}=3.9 \cdot 10^{11} \mathrm{~W} / \mathrm{m}^{2}$.

Fig. 2 shows measurement results for calculated logarithm of $\mathbf{E}_{\uparrow S}$ (forward Stokes component energy) to $\mathbf{E}_{\downarrow S}$ (backward Stokes component energy) ratio with respect to exciting radiation energy $\mathbf{E}_{L 0}$. Energy values are given considering losses in the cuvette windows and all optical elements.

For the maximum energy $\mathbf{E}_{L 0}=310 \mathrm{~mJ}$, the average values were $\mathbf{E}_{\uparrow S}=220 \mathrm{~mJ}, \mathbf{E}_{\downarrow S}=1.1 \mathrm{~mJ}$, and $\operatorname{Lg}\left[\mathbf{E}_{\uparrow S} / \mathbf{E}_{\downarrow S}\right] \approx 2.3$. Thus, the energy asymmetry coefficient reached $k_{\uparrow \downarrow \mathbf{E}}=\mathbf{E}_{\uparrow S} / \mathbf{E}_{\downarrow S} \approx 200$. With $\mathbf{E}_{L 0}$ decrease the forward SRS energy decreased faster. For energy $\mathbf{E}_{L 0}=70 \mathrm{~mJ}$, average values of $\mathbf{E}_{\uparrow S}$ and $\mathbf{E}_{\downarrow S}$ were $\sim 0.25 \mathrm{~mJ}\left(k_{\uparrow \downarrow \mathbf{E}} \approx 1\right)$.

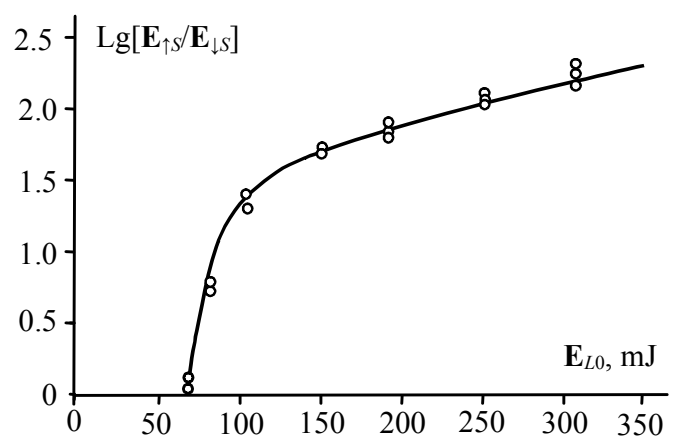

Fig. 2. Experimental dependence of $\mathbf{E}_{\uparrow S}$ to $\mathbf{E}_{\downarrow S}$ ratio on the laser pulses energy $\mathbf{E}_{L 0}$.

\section{Theoretical study}

It was supposed that the laser beam, Stokes component, and anti-Stokes component propagate along $z$ axis, and phase matching conditions are achieved, and that only the Stokes component propagates in the backward direction, since the phase matching of anti-Stokes component is impossible. We've numerically solved the coupled system of equations for the electric field 
amplitudes $E_{L}$ of laser wave and fields $E_{S \uparrow}, E_{S \downarrow}, E_{A}$ of forward Stokes, backward Stokes, and anti-Stokes components, accordingly:

$$
\left\{\begin{aligned}
\frac{d E_{L}}{d z}= & -g_{L}^{\prime} E_{L}\left(E_{S \uparrow}\right)^{2}-g_{L}^{\prime} E_{L}\left(E_{S \downarrow}\right)^{2} \\
& -2 g_{L}^{\prime \prime} E_{L} E_{S \uparrow} E_{A}+g_{L} E_{L}\left(E_{A}\right)^{2} ; \\
\frac{d E_{S \uparrow}}{d z}= & g_{S} E_{S \uparrow}\left(E_{L}\right)^{2}+g_{S}^{\prime \prime} E_{A}\left(E_{L}\right)^{2} ; \\
\frac{d E_{A}}{d z}= & -g_{A}^{\prime} E_{A}\left(E_{L}\right)^{2}+g_{A}^{\prime \prime} E_{S \uparrow}\left(E_{L}\right)^{2} ; \\
\frac{d E_{S \downarrow}}{d z}= & -g_{S}^{\prime} E_{S \downarrow}\left(E_{L}\right)^{2} .
\end{aligned}\right.
$$

If wave frequencies are distant from electronic resonances, refractive indices at laser radiation frequency $\omega_{L}$, Stokes $\omega_{S}$, and anti-Stokes $\omega_{A}$ frequencies are almost equal. By neglecting the nonresonant cubic susceptibility factors in (1), the following equations are valid for $g$ :

$$
\begin{aligned}
& g_{S} / \omega_{S}=g_{L} / \omega_{L}=g_{A} / \omega_{A}, \\
& g_{S}=g_{S}^{\prime}=g_{S}^{\prime \prime}, \quad g_{L}=g_{L}^{\prime}=g_{L}^{\prime \prime}, \quad g_{A}^{\prime}=g_{A}^{\prime \prime} .
\end{aligned}
$$

For benzene, $g_{S}=2.79 \cdot 10^{-14} \mathrm{~m} / \mathrm{V}^{2}[5]$.

The values $E_{S \uparrow}(z=0)=E_{S \downarrow}(z=L)=2 \cdot 10^{4} \mathrm{~V} / \mathrm{m}$ were used for forward Stokes component field $E_{S \uparrow}(z=0)$ at the cuvette inlet and backward Stokes component field $E_{S \downarrow}(z=L)$ at the cuvette outlet, thus providing initial symmetry and efficient generation of forward Stokes components, which are comparable to experimental results at the maximum calculated field of laser radiation $E_{L}(z=0)=1.4 \cdot 10^{7} \mathrm{~V} / \mathrm{m}$. The anti-Stokes field $E_{A}(z=0)$ was considered to be zero. The medium length $L$ was $1 \mathrm{~m}$.

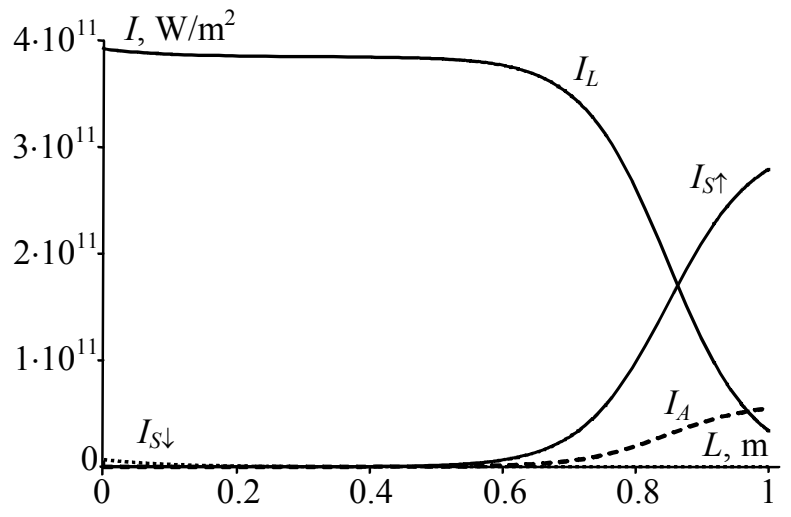

Fig. 3. Radiation intensities in medium considering parametric process in case of $I_{L 0}=3.9 \cdot 10^{11} \mathrm{~W} / \mathrm{m}^{2}$.
The intensity of corresponding components $\left(I_{L}, I_{S \uparrow}\right.$, $\left.I_{S \downarrow}, I_{A}\right)$ was calculated using the electric field amplitude:

$I=0.5 \varepsilon_{0} c n E^{2} \approx 2 \cdot 10^{-3}\left[\mathrm{~W} / \mathrm{V}^{2}\right](E[\mathrm{~W} / \mathrm{m}])^{2}$,

where $\varepsilon_{0}$ is the dielectric constant. Under the boundary conditions, it is $I_{S \uparrow}(z=0)=I_{S \downarrow}(z=0)=8 \cdot 10^{5} \mathrm{~W} / \mathrm{m}^{2}$.

Ignoring the parametric process $2 \hbar \omega_{L}=\hbar \omega_{S}+\hbar \omega_{A}$ in (1) leads to symmetric indicatrix. If parametric process is considered, the forward Stokes component reaches a much higher intensity (Fig. 3). The anti-Stokes component is also observed at the medium output.

Dependence of Stokes component indicatrix asymmetry coefficient (by intensity) $k_{\uparrow \downarrow I}=$ $I_{S \downarrow}(z=L) / I_{S \uparrow}(z=0)$ on the output laser radiation intensity is shown in Fig. 4 in logarithmic scale.

In the given range of laser radiation intensities, $k_{\uparrow \downarrow I}$ varies from 1 to 49 . At $I_{L 0}=3.9 \cdot 10^{11} \mathrm{~W} / \mathrm{m}^{2}\left(E_{L 0}=\right.$ $\left.1.4 \cdot 10^{7} \mathrm{~V} / \mathrm{m}\right)$, we obtain $k_{\uparrow \downarrow I}=40\left(\operatorname{Lg}\left[k_{\uparrow \downarrow I}\right]=1.6\right)$.

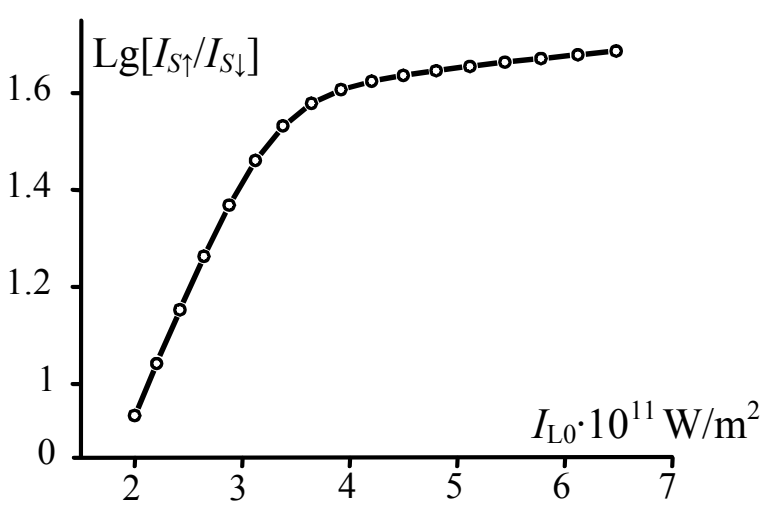

Fig. 4. Theoretical dependence of $I_{S \uparrow}$ (forward SRS Stokes component intensity) to $I_{\downarrow}$ (backward component intensity at $z=0)$ ratio on laser intensity $I_{L}(z=0)$ at $L=1 \mathrm{~m}$.

\section{Conclusions}

The main result of this research is that the predominance of the forward SRS over backward scattering can be caused by the influence of Stokes/anti-Stokes parametric process. The result presents a contrast to the classical notions [5] that do not provide sufficient experimental and theoretical reasoning and, in general, indicates a reverse asymmetry.

The competition between forward and backward scattering processes is important. Stokes/anti-Stokes parametric process provides additional photons to the forward SRS Stokes component. This excess of photons compared to a backward Stokes component increases due to combinational mechanism of stimulated scattering during further propagation of waves along the medium. Thus, during competition in generation of forward and backward Stokes components, the process of forward SRS takes significant advantage.

The calculated logarithm of the scattering 
asymmetry coefficient by intensity in similar experimental conditions reaches the value close to 1.6 at maximal laser radiation intensities. Experimental values of the asymmetry coefficient logarithm by energy are much higher and reach $\sim 2.3$.

Differences of experimental and theoretical results might have appeared due to neglection of radiation intensity change during the laser pulse. However, the more important is presence of self-phase modulation, despite the fact that benzene does not reveal distinct selffocusing properties.

\section{References}

1. G. Placzek, Rayleigh Scattering and the Raman Effect. Moscow-Leningrad, Gostekhizdat, 1935 (in Russian).

2. I.I. Kondilenko, P.A. Korotkov, On the asymmetry of the Raman scattering indicatrix // Optika $i$ Spektroscopiya, 24, p. 466-468 (1968), in Russian.

3. N. Bloembergen and Y.R. Shen, Coupling between vibrations and light waves in Raman media // Phys. Rev. Lett. 12(18), p. 504-507 (1964).
4. P.D. Maker and R.W. Terhune, Study of optical effects due to an induced polarization third order in electric field strength // Phys. Rev. 137(3A), p. 801818 (1965).

5. Y.R. Shen, The Principles of Nonlinear Optics. New York, Wiley, 1984.

6. V.V. Kuzmin, Disruption of phase matching in stimulated light scattering // Trudy FIAN, 207, p. 339 (1991), in Russian.

7. A.V. Kaczynski, G.G. Kotaev, I.V. Pilipovich, Competition between backward and forward SRS and compression of picosecond pulses // Kvantovaya Elektronika, 19(6), p. 550-553 (1992), in Russian.

8. Iu. E. D'yakov, S.Iu. Nikitin, On the interaction and competition between forward and backward SRS scattering // Kvantovaya Elektronika, 9(6), p. 12581261 (1982), in Russian.

9. G.S. Felinskiy, P.A. Korotkov, Simulation of multiwave pumped fiber Raman amplifiers // Semiconductor Physics, Quantum Electronics \& Optoelectronics 9(3), p. 35-39 (2006). 